\title{
La ecoaldea Los Guindales: escuela intensiva para la conservación de la cultura y la vida de las zonas rurales
}

África Rodríguez Nieves | trabajadora social y naturópata, cofundadora de la Ecoaldea Los Guindales

Loris Carboni | carpintero de armar y bio-constructor, cofundador de la Ecoaldea

Los Guindales

URL de la contribución <www.iaph.es/revistaph/index.php/revistaph/article/view/4510>

\section{RESUMEN}

La ecoaldea Los Guindales es un plan grupal que nace hace 15 años y, a lo largo del tiempo, hemos generado dos proyectos interdependientes: la casa Los Guindales y la escuela de bioconstrucción Los Guindales, con el sueño común de cuidar la Madre Tierra y participar en la transformación de esta sociedad hacia un modelo más humano donde cuidar la vida sea la prioridad. Compartimos un ecosistema natural, una finca en un bosque comestible en el valle del Genal, en la localidad de Algatocín, en la serranía de Ronda, Málaga; un conjunto de valores; y el compromiso con la realidad social a la que pertenecemos, que nos lleva a una continua reflexión sobre los valores ancestrales que permitieron, durante miles de años, que los seres humanos vivieran en armonía con la Naturaleza, cubriendo sus necesidades básicas. En una continua integración y puesta en práctica de los valores que guían nuestros propósitos: compromiso con la Tierra y las futuras generaciones, revalorización de los saberes campesinos, madurez emocional, ayuda mutua, solidaridad, autonomía y autogestión.

\section{Palabras clave}

Algatocín (Málaga) | Autogestión | Bioconstrucción | Despoblación | Ecoaldeas | Los Guindales | Neorrurales | 


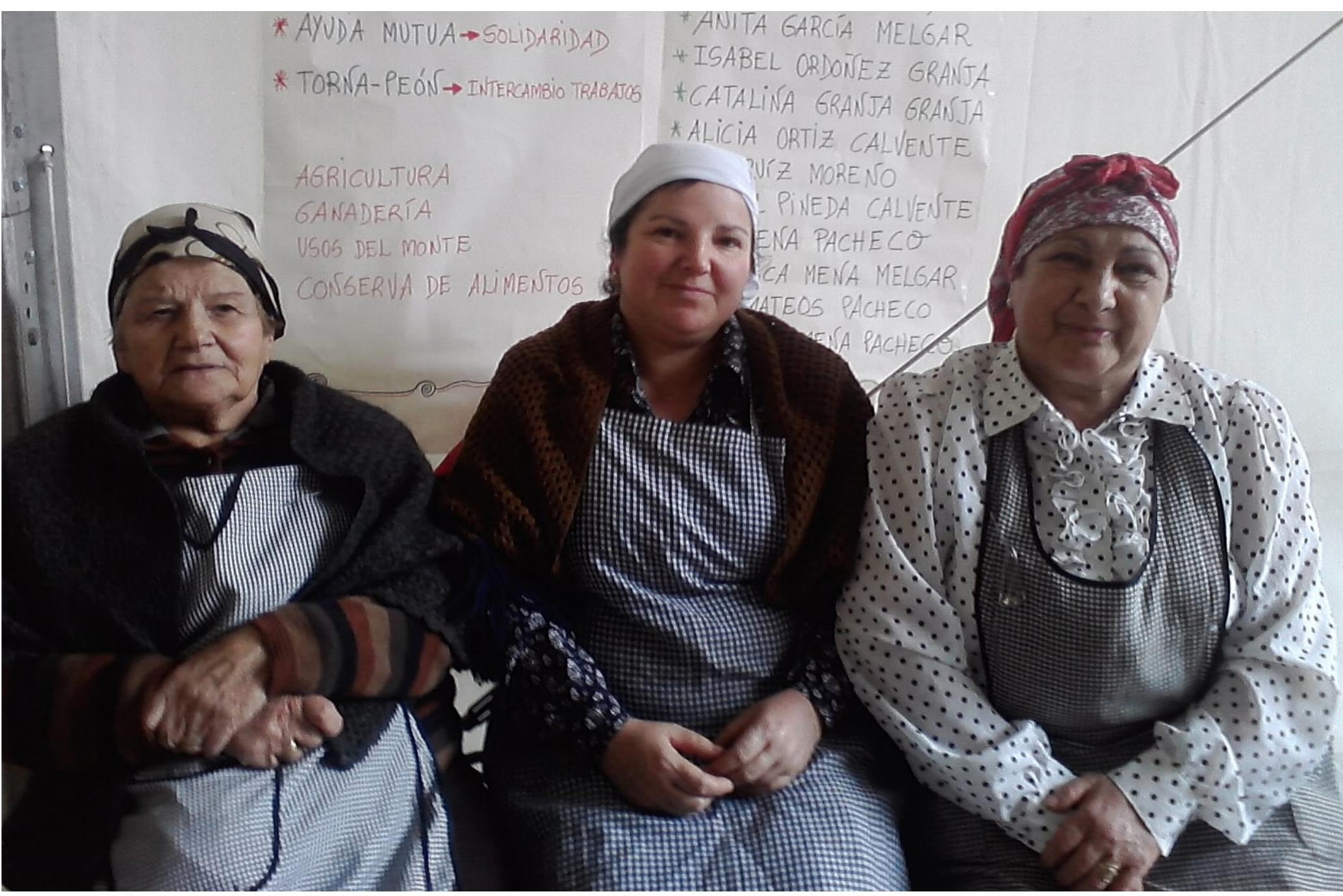

Proyecto Tradición oral con mayores (Algatocín) | foto Rosa Pacheco Navarro 
La ecoaldea Los Guindales es un proyecto grupal que nace hace 15 años y, a lo largo del tiempo, hemos generado dos proyectos interdependientes: la casa Los Guindales y la escuela de bioconstrucción Los Guindales, con el sueño común de cuidar la madre tierra y participar en la transformación de esta sociedad hacia un modelo más humano donde cuidar la vida sea la prioridad. Compartimos un ecosistema natural, una finca en un bosque comestible en el valle del Genal, en la localidad de Algatocín, en la serranía de Ronda, Málaga; un conjunto de valores; y el compromiso con la realidad social a la que pertenecemos, que nos lleva a una continua reflexión sobre los principios ancestrales que permitieron, durante miles de años, que los seres humanos vivieran en armonía con la naturaleza, cubriendo sus necesidades básicas. Trabajamos por una continua integración y puesta en práctica de los valores que guían nuestros propósitos: compromiso con la Tierra y las futuras generaciones, revalorización de los saberes campesinos, madurez emocional, ayuda mutua, solidaridad, autonomía y autogestión.

La gestión de esta finca la desarrollamos a través de nuestro propio diseño de permacultura, entendiéndola como un conjunto de saberes que permita que nuestras vidas sean social, económica y ecológicamente sostenibles. La permacultura la entendemos como una cultura que permanece en el tiempo, específica y única en cada territorio, pero que comparte en general unos elementos esenciales para la vida como el agua, el alimento, el cobijo, la salud, las relaciones interpersonales y los poderes de la naturaleza. Al mismo tiempo que integra las innovaciones propias de la realidad social, como las nuevas tecnologías aplicada a estos diferentes ámbitos.

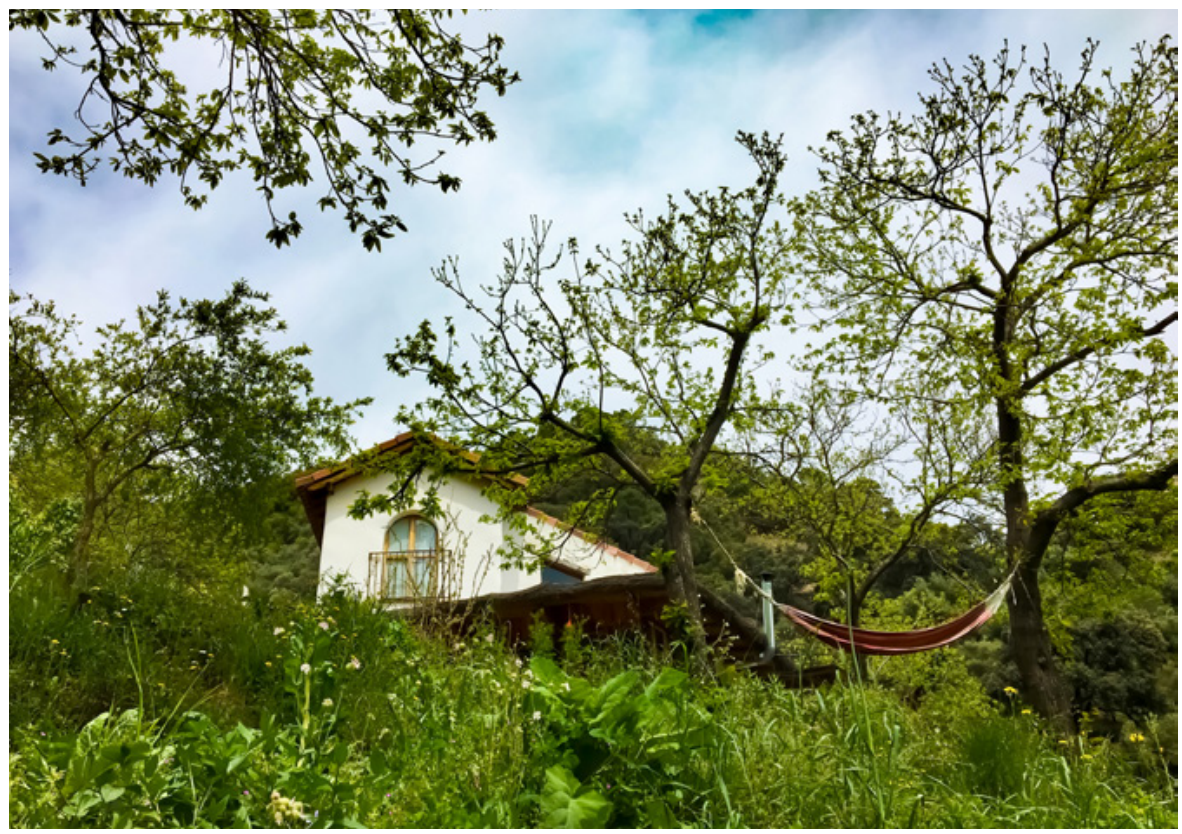


La autogestión es la filosofía que guía nuestra forma de desarrollar y cubrir una parte de nuestras necesidades básicas: autoconstrucción de los hogares, huertas familiares, gallinero, manejo del bosque de castaños y silvicultura, sistemas de abastecimiento de agua y depuración, arreglos de caminos, medicina natural (fitoterapia), etc. Mientras que otra parte de nuestra economía, ya que no somos totalmente independientes del sistema, ha surgido con intercambios de ayuda mutua y generando economía propia. Esto nos lleva a soportar jornadas intensas de trabajo, con muchas responsabilidades, propias de la vida en el campo; una realidad muy diferente a la que observamos en una gran mayoría de personas que tienen una visión idílica de este tipo de vida, la cual conlleva muchas renuncias y una gran disciplina. Lo que nos hace valorar aún más la economía de subsistencia que desarrollan nuestros mayores en las zonas rurales.

Entendemos el grupo o comunidad como un modelo alternativo a la familia tradicional, ya que somos seres sociales y para compartir las tareas del día a día. Para ello nos hemos organizado creando hogares y espacios compartidos de convivencia, siendo interdependientes entre nosotros y con el sistema, frente a la idea del individualismo y la dependencia al consumo que genera el modelo capitalista actual.

Cada uno de los proyectos que integra la ecoaldea gira en torno a la idea de la conservación del patrimonio cultural como propuestas para combatir el despoblamiento rural y ofrecer un modelo de vida sostenible.

La Casa de los Guindales se centra en: Investigación y estudio de los saberes campesinos de las diferentes culturas de los pueblos originarios y, específicamente, de los conocimientos de este territorio en el sur de Andalucía; Reflexión e integración en nuestras vidas de los valores humanos que nutren una relación en armonía con uno mismo (autoconocimiento), con los demás (habilidades psicosociales) y con el entorno (compromiso con el planeta Tierra y las futuras generaciones), siguiendo un diseño de permacultura; $Y$ Apertura de nuestro proyecto a personas que viven principalmente en espacios urbanos a través de visitas, participación en talleres y experiencias de voluntariado de aprendizaje, así como inspiración para otras personas motivadas para la creación de proyectos grupales o familiares en el medio rural de forma sostenible.

\section{SALVAGUARDA DEL PATRIMONIO INMATERIAL}

La Escuela de Bioconstrucción Los Guindales tiene entre sus fundamentos la conservación del patrimonio cultural inmaterial, además de dar respuestas ante la emergencia medioambiental y de salud de las personas en las viviendas. El patrimonio cultural inmaterial infunde un sentimiento de identidad, se 


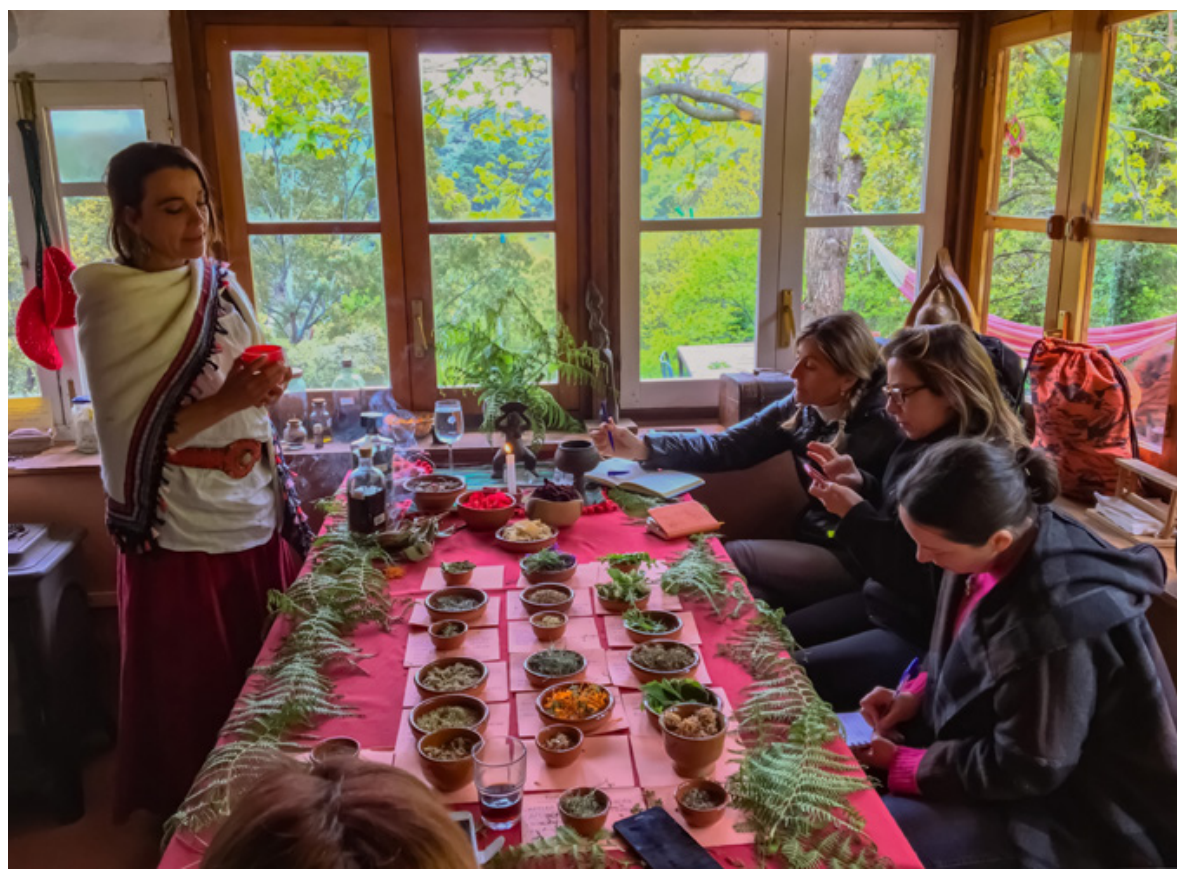

Taller de autogestión de la salud: Hierbas medicinales andaluzas, identificación, propiedades y usos, realizado en la Casa Los Guindales | foto Gabriel Carrillo Calderón transmite de generación en generación y es recreado constantemente por las comunidades. En particular, nos centramos en las técnicas artesanales tradicionales relacionadas no sólo con la construcción, sino con la agricultura y la silvicultura, casi desaparecidas, poniéndolas en valor frente a las formas actuales de construcción de baja eficiencia energética constructiva y climática, que no guardan equilibrio con el entorno. Nos hemos formados como bio-constructores, en estas tierras andaluzas, gracias a otras experiencias y a personas que han decidido compartir humildemente su sabiduría.

Y es que el patrimonio cultural inmaterial de este territorio está salvaguardado por las personas mayores del lugar, siendo un ejemplo vivo de las diferentes formas de gestión del medio rural basado en un modelo de subsistencia en una sociedad agrícola -desafortunadamente en decrecimiento-. A lo largo de muchos años, este lugar fue sufriendo diferentes migraciones a otros lugares urbanos, principalmente, de mayor facilidad de acceso a los modelos de producción propios del capitalismo; que ocasionó una ruptura o brecha generacional sobre la transmisión del legado cultural, unido a los cambios en el modo de aprendizaje y acceso al conocimiento debido al desarrollo tecnológico.

El grupo inicial que comenzamos este proyecto nos conocimos en otro proyecto de permacultura. Procedíamos de zonas urbanas, de diferentes lugares de origen, varias nacionalidades y de diversos ámbitos de activismo social, y compartíamos un gran entusiasmo de poder "crear" el modo de vida en el que creíamos en un entorno rural. Éramos seis mujeres, un hombre y 
cuatro menores. Estuvimos desarrollando un trabajo grupal durante un año para ir diseñando nuestra futura idea, a la vez que nos fortalecíamos como grupo, favoreciendo la cohesión grupal utilizando las diferentes herramientas de la facilitación grupal (comunicación no violenta, toma de decisiones por consenso, resolución de conflictos, etc). Estuvimos visitando muchas fincas con la idea de encontrar la tierra más adecuada a nuestro fin. Al llegar a este valle decidimos alquilar una finca para conocer mejor esta zona y seguir definiendo el proyecto. Un año después compramos la finca Los Guindales. Al instalarnos en el valle nuestra primera motivación fue acercarnos a la personas mayores, visitándolas en sus campos y compartiendo con ellas todas nuestras dudas e inquietudes. Nos recibían con mucha hospitalidad y alegría al comprobar cómo un grupo de jóvenes se interesaba por sus quehaceres diarios, el desenvolvimiento de sus vidas y el manejo de las diferentes artes campesinas; nos veían llenos de ilusiones para desarrollar en su territorio nuestro sueño. La escucha y observación eran nuestras aliadas para impregnarnos de tanta sabiduría.

La Casa de los Guindales comenzó desarrollando diferentes proyectos sociales a través de la dinamización socio-cultural, utilizando la metodología de Investigación Acción Participativa, en colaboración con asociaciones y corporaciones locales, de donde surgió la idea de la recopilación y revalorización de saberes campesinos, así como, de visibilizar la "economía invisible" de la mujer rural y su labor como guardianas y dinamizadoras de la cultura local. A partir de ello, se elaboraron diferentes trabajos etnográficos en relación con cada una de las artes y oficios antiguos (hoy en día muchos de ellos desaparecidos), que han quedado reflejados en diferentes materiales y publicaciones como calendarios agrícolas, recetarios y publicaciones de "Historias de Vidas". Además, se han llevado a cabo talleres de artesanía con corcho, degustaciones de platos típicos, teatro terapéutico y representaciones teatrales en las jornadas de Semana Cultural, recreaciones de antiguos oficios y actividades domésticas, entre otras.

Nuestra acción y compromiso nos hace participar a través de conferencias y talleres en diferentes ámbitos de la sociedad: centros educativos (asociaciones de AMPAS y Centro de Profesorado -CEP), asociaciones de mujeres, foros de discusión y participación con otras entidades dedicadas a la ecología, bioconstrucción y salud, como la Red ibérica de Ecoaldeas y otras plataformas. En la finca ofrecemos diferentes posibilidades como experiencia vivencial, compartiendo programas de voluntariado de aprendizaje, alojamientos y talleres. Abordamos diferentes temas de interés como hábitos saludables, fitoterapia del lugar, cosmética natural, educación emocional, desarrollo personal y bioconstrucción.

En la Escuela de Bioconstrucción investigamos y estudiamos las técnicas de construcción antiguas utilizadas en muchos lugares del mundo, que mues-

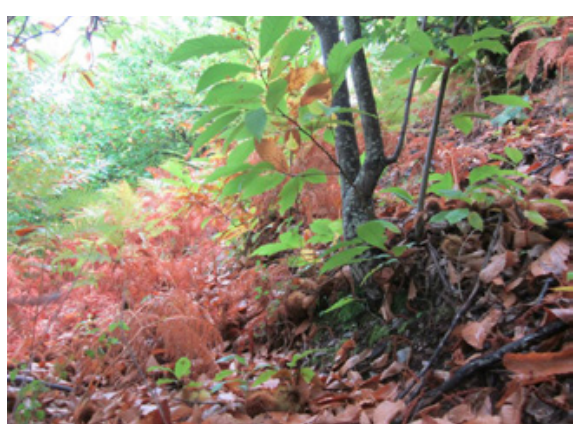

Bosque de castaños



Cría de aves de corral | fotos Ecoaldea Los Guindales 


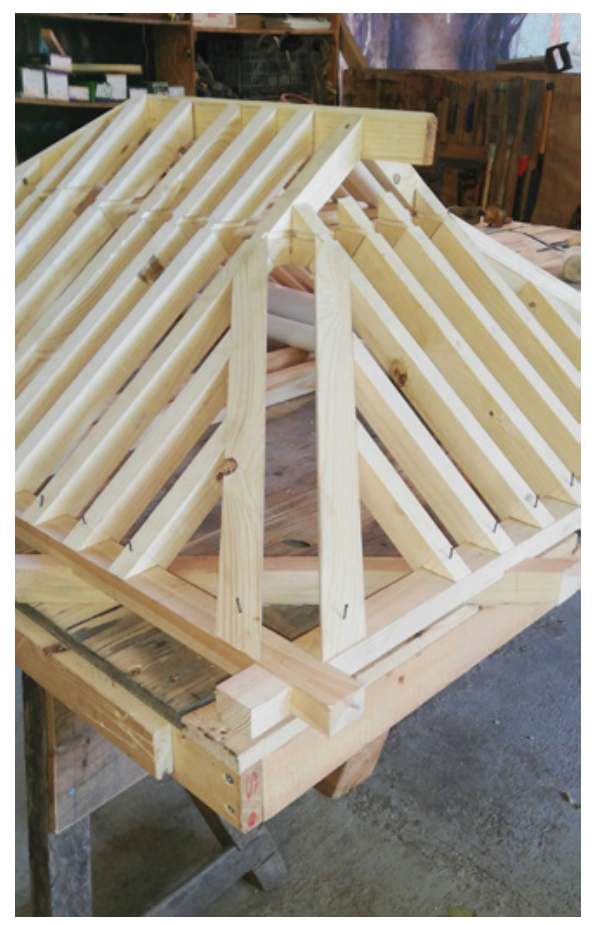

A la izquierda, maqueta de techo de pares y nudillos; a la derecha, construcción en carpintería de armar. Escuela de Bioconstrucción | fotos Ecoaldea Los Guindales

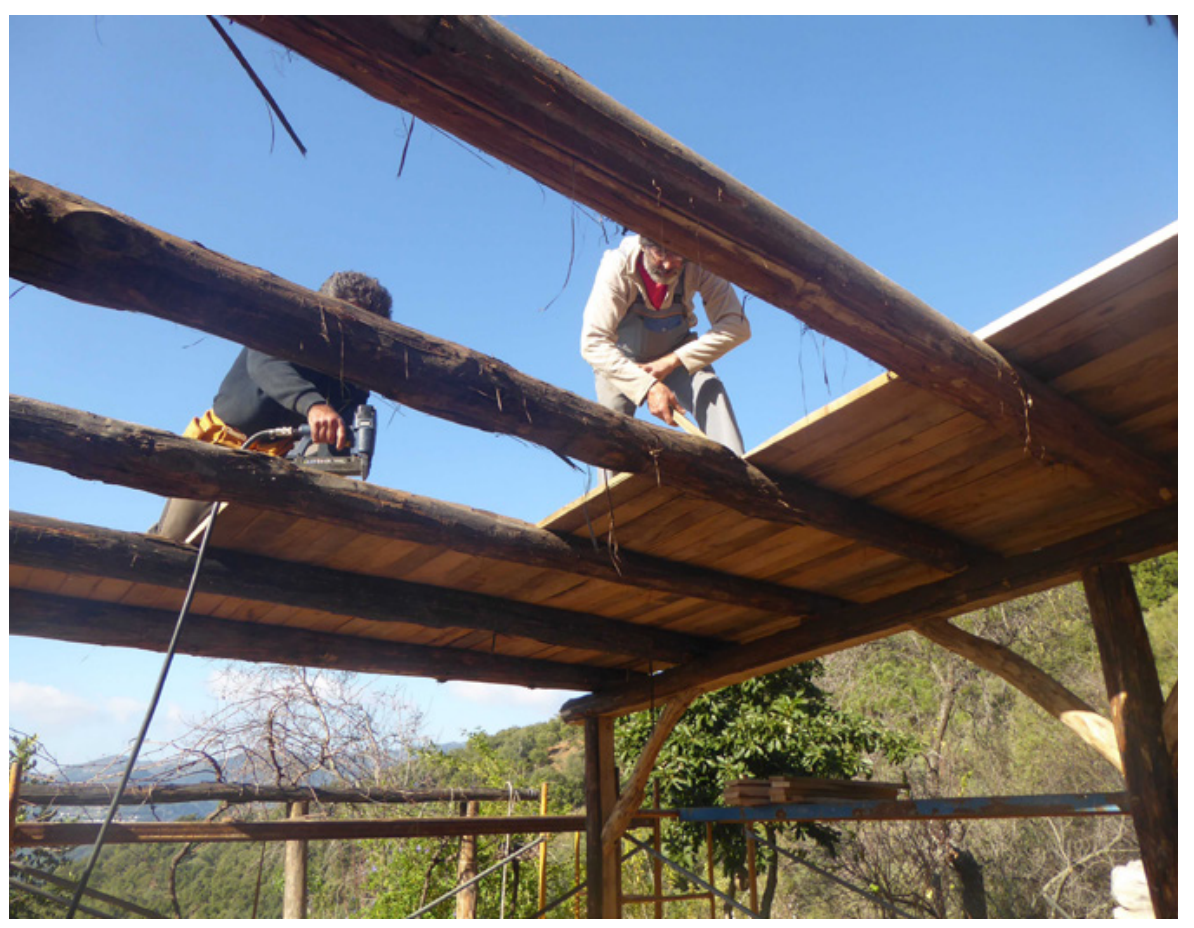

tran múltiples soluciones a la necesidad humana primordial que es el cobijo y ofrecen una gran cantidad de posibilidades constructivas con los materiales básicos comunes a todas ellas: la tierra, la piedra, la madera y las fibras vegetales; estos cuatro materiales, sus múltiples variedades y tipologías junto con el conocimiento, abren una puerta a un mundo fabuloso por re-descubrir. Nuestro recorrido como escuela -ofreciendo aprendizaje, el estudio, investigación y práctica- se dirige principalmente a la carpintería de armar tradicional, oficio que queremos conservar y cuidar; y nos motiva saber que en muchas partes del mundo hay muchos oficiales y artesanos usando este material tan noble, fuerte y duradero, como es la madera. También hemos impulsado diferentes iniciativas de investigación, aprendizaje y construcción con la cal, como material básico en la construcción tradicional. Históricamente, a mediados del siglo XX, hubo un apogeo de la fabricación artesanal de la cal en la comarca del Genal. Solo en el término de Algatocín había más de 100 caleras funcionando.

La bioconstrucción como concepto contemporáneo viene conformándose desde hace unas décadas. Nace como una disciplina nueva, clara e integradora que permite cumplir objetivos comunes a campos tan diferentes como el de las tecnologías sostenibles, ecología, arquitectura bioclimática o la salud pública, con la coherencia y la visión de futuro imprescindibles para el desarrollo del planeamiento urbanístico, la arquitectura y la construcción en general. 
Por otro lado, tenemos la motivación de compartir con otras personas nuestras experiencias, saberes y espíritu creativo; esto nos ha llevado a desarrollar un "modelo económico" múltiple, abierto y flexible. El saber tiene que ser compartido, para que no se olvide, para que si algún día el petróleo se acaba, podamos seguir utilizando nuestras habilidades artesanales en armonía con la Tierra. Una de las dificultades que nos hemos encontrado para poder transmitir estos saberes a nivel oficial a través de una escuela taller dirigida a jóvenes es la negativa a nuestra solicitud por parte de la administración pública. Esperamos que algún día se contemple esta posibilidad para formar a nivel profesional a jóvenes en este sector y se puedan recuperar estos oficios.

La mayoría de las personas que en todos estos años hemos participado en este proyecto crecimos en entornos urbanos y sentimos en su día "la llamada" a una vida con la naturaleza, debido a la deshumanización de la vida en las ciudades y a las actuaciones erróneas de gobiernos y empresas a nivel social y medioambiental.

Las principales dificultades que nos hemos encontrado para nuestra inclusión en este territorio han sido el acceso a la tierra, el desarraigo con nuestras familias de origen y cierta desconfianza de los lugareños a nuevas formas de concebir el desarrollo humano y económico en relación con el medio. Por otra parte también hemos tenido el apoyo y aceptación de las corporaciones locales, aunque para ello hemos tenido que demostrar nuestra máxima profesionalidad en los diferentes ámbitos en que hemos colaborado. Se han credo fuertes lazos de amistad y vecindad con las personas autóctonas. Respetamos las tradiciones y fiestas religiosas de este lugar, y participamos y nos involucramos en muchas de sus otras actividades culturales y educativas.

Consideramos que la vuelta al campo es un camino que enriquece socialmente a todos los niveles, generando intercambios y activando pequeñas economías tanto tradicionales como innovadoras y revalorizando la vida en el medio rural frente al problema de la despoblación rural que desde hace mucho tiempo sufren estos lugares. Creemos que una solución práctica al despoblamiento rural es la concienciación y fomento de la gestión de los recursos naturales de la zona: huertas ecológicas para la venta en ecotiendas y grupos de consumo, apicultura, elaboración de compost orgánico, saca de la corcha, desbroce y mantenimiento de carreteras y cauces del río, recuperación de veredas y cañadas reales, turismo sostenible, pastoreo, leyes que permitan la artesanía alimentaria, cosmética natural y preparados herbales, etc.

Creemos que estamos siendo un referente, tanto para los lugareños como para los visitantes, de valores que respetan y cuidan al ser humano desde



Feria gastronómica. Destilación de aceites esenciales

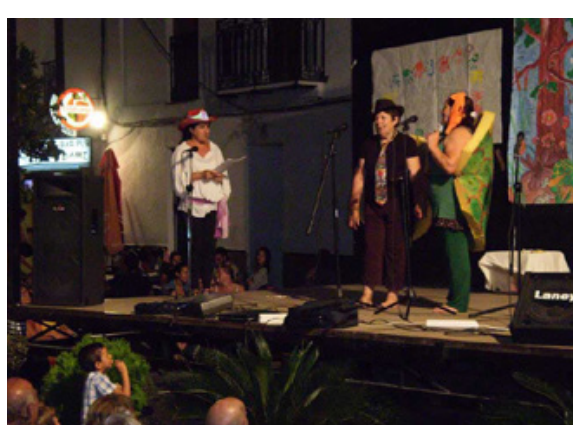

Representación teatral Asociación de mujeres Banu-Rabah | fotos Ayuntamiento de Benarrabá 


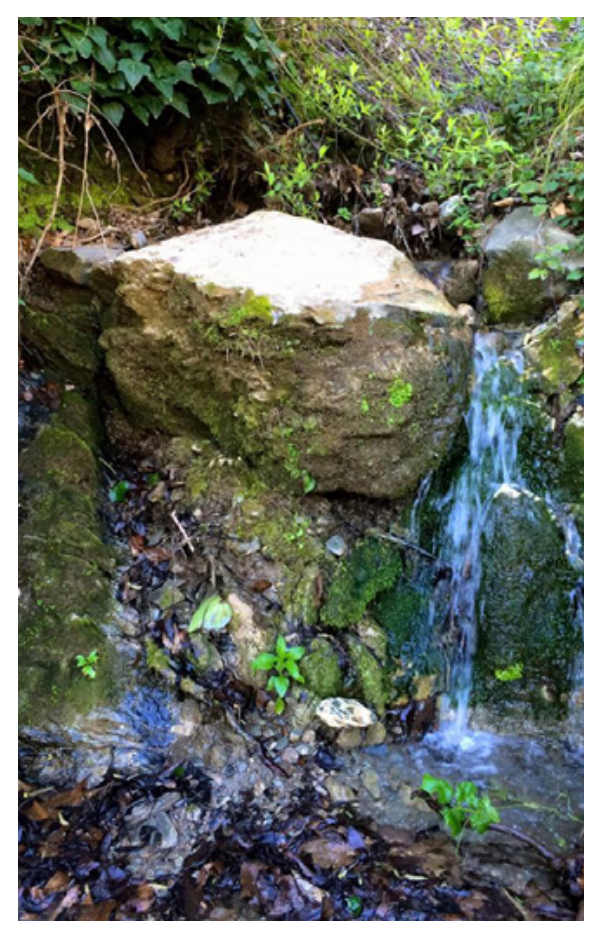

Arroyo y amanecer en la finca Los Guindales | fotos Ecoaldea Los Guindales

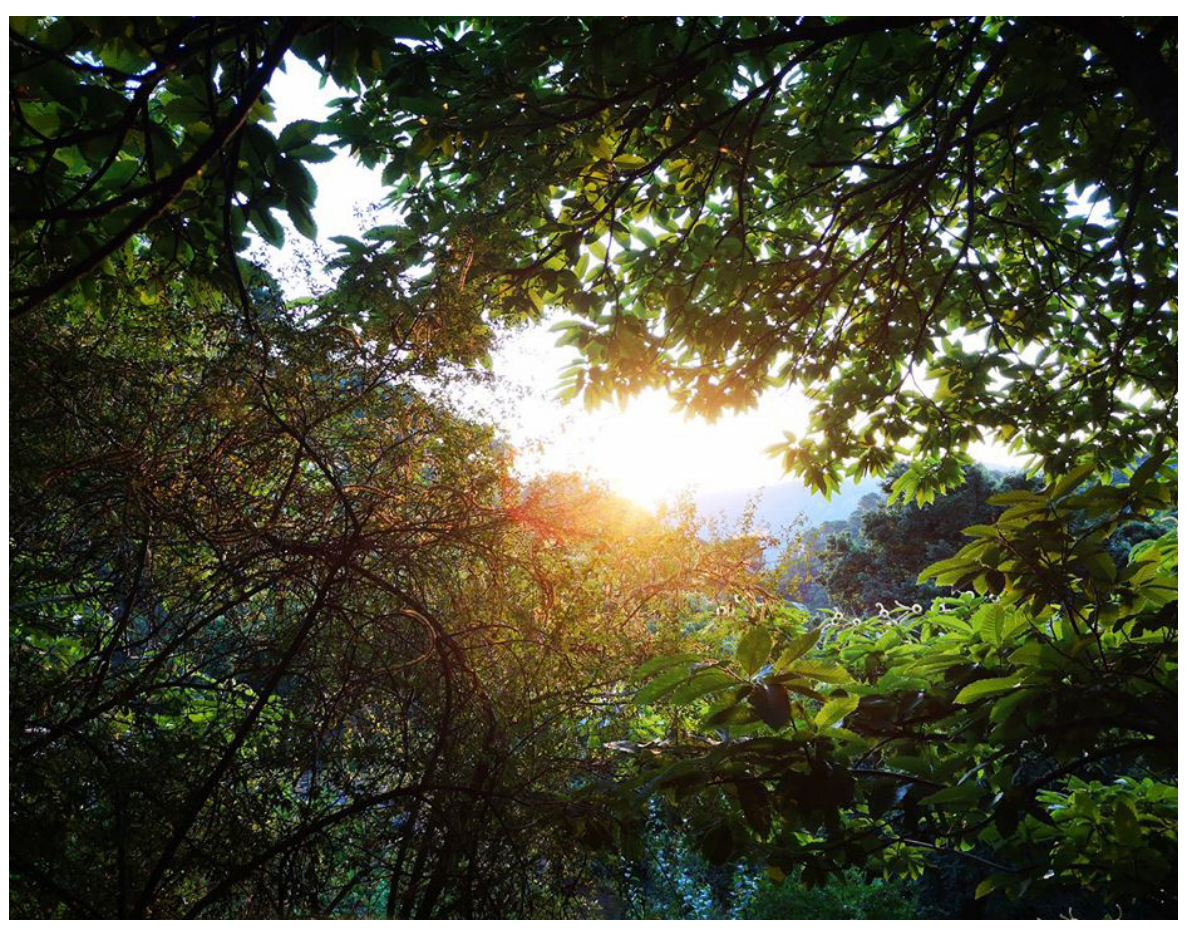

una visión de conciencia de uno mismo, de nuestras relaciones con los demás y con el medio.

Algunos de nuestros hijos e hijas han nacido en este hermoso valle y están integradas en el ámbito escolar y en sus relaciones sociales. Es un enriquecimiento cultural el poder compartir entre ellos diferentes realidades familiares y estilos de vida.

Haciendo un balance de nuestra presencia en este lugar, podemos clasificarla como satisfactoria, ya que creemos que con nuestro ejemplo estamos mostrando que es posible otra forma de vida. Hemos conseguido una finca sostenible, un flujo constante de personas que contactan o visitan con diferentes temas de interés y motivaciones, una economía estable basada en pequeñas economías, un modelo de turismo sostenible integrado en nuestro día a día, colaboración con diferentes redes, respeto mutuo y participación en la vida del pueblo, motivación para otras personas y, lo que consideramos más importante, este espacio vivo como escuela intensiva de los valores que permiten que la cultura de un lugar se pueda conservar y ayudar a que los pueblos y zonas rurales se mantengan vivos.

Nuestra visión futura es seguir trabajando a favor de la transmisión de saberes, cuidar la vida de la tierra fomentando la biodiversidad del bosque y poder seguir creciendo como personas. 
Nos preocupa la gran estrategia mundial destructiva que se está moviendo contra la vida y los valores que emergen en contra de la multiculturalidad. Somos conscientes de que la forma de vida actual es contra-natura y nos enfrentamos cada día a muchos retos e incoherencias; es inevitable el impacto que creamos en la naturaleza y mantenernos como parte de esta sociedad nos obliga a aceptar muchas de las necesidades que nos impone para hacer efectiva dicha participación, por ejemplo, el uso de las nuevas tecnologías y la burocracia administrativa.

Sentimos que solo somos un granito de arena en este mundo, resistiendo en este lugar y con el corazón contento seguimos creyendo que, en palabras de Eduardo Galeano: "Mucha gente pequeña, en lugares pequeños, haciendo cosas pequeñas, puede cambiar el mundo". 